\section{Comparison of pulsatile ocular blood flow in Indians and Europeans}

\author{
Abstract \\ Purpose To compare pulsatile ocular blood \\ flow (POBF) in Europeans and Indians and \\ provide reference values for a group of healthy \\ Indians. \\ Patients and Methods Measurement with the \\ POBF Tonograph was performed on healthy \\ Indian subjects in India $(n=252)$. A further 80 \\ subjects (40 of Indian descent and 40 \\ Europeans) underwent measurements in \\ Cambridge, England. The instrument used \\ for measurement was the same for both the \\ studies. \\ Results The mean POBF in the Indians in \\ India was found to be $1176 \mu \mathrm{l} / \mathrm{min}$. The mean \\ POBF value in the Europeans was found to be \\ $1033 \mu \mathrm{l} / \mathrm{min}$ and that for Indians in England \\ was $1061 \mu \mathrm{l} / \mathrm{min}$. The difference between the \\ POBF within groups was significant (one-way \\ ANOVA $P<0.05$ ) with the POBF of Indians in \\ India being higher than Europeans and \\ Indians in the UK. The difference between the \\ Europeans and Indians in the UK did not \\ reach statistical significance. \\ Conclusions POBF values in Indians living \\ in India were found to be considerably higher \\ than the previously published normal value of \\ $650 \mu \mathrm{l} / \mathrm{min}$ in European studies and other \\ studies for other racial groups. The reason for \\ this apparent difference may be instrument- \\ related rather than genetic because such a \\ large difference was not observed when \\ a comparison was performed in the UK. \\ In addition, the results for both groups in our \\ comparative study were still considerably \\ higher than reported in previous studies. \\ The POBF of Indians in India is slightly \\ higher than the POBF of people of Indian \\ ethnic origin in England. \\ Eye (2005) 19, 1163-1168. doi:10.1038/sj.eye.6701710; \\ published online 24 September 2004
}

P Gunvant1', M Baskaran², L Vijaya², BC Hansen' IS Joseph², RJ Watkins ${ }^{3}$, DC Broadway ${ }^{4}$ and DJ O'Leary ${ }^{5}$
Keywords: pulsatile ocular blood flow; intraocular pressure; Indians; Europeans; glaucoma

\section{Introduction}

The Pulsatile Ocular Blood Flow (POBF) Tonograph, now called the Ocular Blood Flow (OBF) Analyzer (Paradigm Medical Industries, Inc., Salt Lake City, UT, USA), is a modified Langham pneumotonometer, which uses an air piston system to applanate the cornea and continuously record the intraocular pressure (IOP) and POBF is calculated. The working principles of a pneumotonometer have been described in detail elsewhere. ${ }^{1}$ Briefly, the blood flow of the choroid is pulsatile in nature. As a bolus of blood enters the choroid during systole there is a change in the volume of the eye, which is observed as an ocular pulse. The ocular pulse can be measured accurately by continuously recording the IOP. The POBF Tonograph also calculates the pulse amplitude and measures the pulse rate. The POBF Tonograph uses IOP and pulse amplitude and estimated values for ocular rigidity, based on the Friedenwald equation, to calculate pulse volume. ${ }^{1}$ The POBF Tonograph uses the slope of the IOP pulse (ie the differential) to calculate the POBF. ${ }^{1,2}$

The equations used and assumptions made in the calculations of POBF have been investigated widely. $^{2-7}$ The measurements have been reported to be reproducible ${ }^{8-10}$ and the theoretical assumptions used for the calculations are acceptable. ${ }^{2}$ Factors which may influence measurement include probe positioning and practitioner experience. ${ }^{9}$

Various conditions such as glaucoma ${ }^{11-15}$ diabetes mellitus, ${ }^{16,17}$ and chorio-retinal lesions ${ }^{18-20}$ have been reported to affect
${ }^{1}$ Department of Psychological and Brain Sciences

University of Louisville Louisville KY, USA

${ }^{2}$ Medical Research

Foundation

Chennai, India

${ }^{3}$ Department of Cellular Biology

Royal Preston Hospital Sharoe Green Lane

Preston, UK

${ }^{4}$ Department of Ophthalmology Norfolk and Norwich University Hospital NHS Trust

Norwich, England

${ }^{5}$ Department of Optometry and Ophthalmic Dispensing Anglia Polytechnic

University

Cambridge, UK

Correspondence: P Gunvant University of Louisville 317, Life Sciences Building Louisville KY 40292, USA

Tel: + 15028524056

Fax: + 15028528904

E-mail: contact@

pinakin-gunvant.com

Received: 17 April 2004 Accepted in revised form: 27 July 2004

Published online: 24 September 2004 
pulsatile ocular blood flow. There is also evidence of racial differences in POBF. Lam et al ${ }^{8}$ reported that POBF in Hong Kong Chinese was lower than reported for other racial groups. They concluded that the high incidence of refractive error (myopes tend to have greater axial length and hence higher ocular volume) in Hong Kong Chinese was the cause for lower values of POBF but could not exclude racial or genetic influences due to the absence of a European control group in the study. As far as we are aware, the reference values of POBF for an Indian population have not been established.

The main aim of the present study was to determine reference values of POBF for a normal clinic sample in India and to compare such normative data with other published values (study 1). Studies have been previously conducted in Europe. ${ }^{9-11,21}$ Their pneumotonometers used a different software version ${ }^{9-11}$ or a different manufacturer ${ }^{21}$ to the one used in the present study. Because study 1 gave unexpected results, a second study was designed to compare the POBF values in Indians in England and Europeans (study 2).

\section{Materials and methods}

\section{Pulsatile ocular blood flow reference values for Indians (study 1)}

In total, 252 subjects (122 males and 130 females) were examined in the outpatient department of the Rural Eye Hospital, Sankara Nethralaya (a unit of the Medical Research Foundation) in Chennai, India.

Each subject underwent a complete ophthalmic examination, including refraction, external eye evaluation with a slitlamp, and dilated retinal evaluation by an ophthalmologist. Subjects with signs of eye disease, known systemic disease, or having a history of any intraocular surgery were not included in the study.

A Goldmann tonometer mounted on a Haag-Streit slitlamp was used to measure IOP throughout the study and an observer (ISJ) performed all the Goldmann applanation tonometry measurements. All the POBF measurements were done with the POBF Tonograph mounted on a slitlamp, by an experienced observer (PG).

Subjects underwent IOP measurement using both the Goldmann applanation tonometer and the POBF

Tonograph. Both the GAT and the POBF Tonograph were calibrated according to the manufacturer's guidelines.

A drop of topical anaesthetic (proxymetacaine $0.5 \%$ ) was used prior to the measurement with the POBF Tonograph and a fluorescein anaesthetic combination (proxymetacaine $0.5 \%$ with fluorescein sodium $0.25 \%$ ) was used prior to the Goldmann applanation tonometer measurement. Subjects were cyclically allocated a serial number in order of attendance. Individuals with an odd serial number underwent measurements with the Goldmann applanation tonometer first and subjects with even serial numbers underwent measurements with the POBF Tonograph first. There was a 15-min interval between the IOP measurements to minimize any possible carry-over effects due to sequential tonometry.

\section{Comparison of pulsatile ocular blood flow values between Indians and Europeans (study 2)}

In all, 80 subjects (40 Indians and 40 European) from the staff and student population of Anglia Polytechnic University were included. Indians were subjects having their ethnic origin entirely from India, Pakistan, or Bangladesh. The Europeans were subjects of their ethnic origins from a variety of European countries (mainly England).

The subjects in both the groups were matched for age, gender, and refraction. The protocols used were identical to those used in India except that Goldmann applanation tonometry was not performed. An experienced observer (PG) performed all the IOP measurements using the POBF Tonograph. The same POBF Tonograph was used in both studies 1 and 2 (POBF Tonograph software version 16.2).

The Medical Research Foundation Ethics Committee and Anglia Polytechnic University Research Ethics Committee approved the studies; the tenets of the Declaration of Helsinki were observed and data management conformed to HIPPA regulations. Written informed consent was obtained from all study participants.

\section{Statistical analysis}

Data were analysed using SPSS 12.0 for Windows. One-way analysis of variance (ANOVA) was used to investigate the differences within groups and between groups for age, mean spherical refraction, intraocular pressure measured using the Goldmann applanation tonometer and other parameters measured by the POBF Tonograph, that is, pulse amplitude, pulse rate, IOP, and POBF (studies 1 and 2). Linear regression analysis was used to investigate the relationship between age and POBF and between refractive error and POBF (study 1). A two-way ANOVA was used to investigate the interaction for ethnic origin and gender with respect to POBF (study 2). A one-way analysis of covariance (ANCOVA) was used to investigate the differences in POBF between groups at different geographical locations with age as the covariate (study 2). 


\section{Results}

Pulsatile ocular blood flow reference values for an Indian (study 1)

Table 1 shows the mean values of age, spherical refraction, IOP measured by the GAT, IOP measured by the POBF Tonograph, pulse amplitude, pulse rate, and POBF for males and females enrolled in the study. Both the male and female groups were similar for age (one-way ANOVA, $F=1.2 ; P>0.05$ ), refraction (one-way ANOVA, $F=1.9 ; P>0.05)$, IOP measured by both by the GAT (one-way ANOVA, $F=1.4 ; P>0.05$ ), and the POBF Tonograph (one-way ANOVA, $F=0.14 ; P>0.05$ ).

For males and females, POBF was independent of age (Linear regression males, $R^{2}=0.002 ; P>0.05$ and females, $\left.R^{2}=0.00001 ; P>0.05\right)$. As expected, there was a significant relationship between mean spherical refractive error and POBF (linear regression, $R^{2}=0.052$; $P<0.001)$. The effect of refractive error on POBF is given by the following regression equation:

$$
\mathrm{POBF}=43.31(\text { mean spherical refraction })+1202.7
$$

Males and females had significant differences in pulse amplitude (one-way ANOVA, $F=10.8 ; P<0.05$ ), pulse rate (one-way analysis of variance, $F=7.39 ; P<0.05$ ), and POBF (one-way ANOVA, $F=24.68 ; P<0.001$ ) with females having significantly higher values than males.

\section{Comparison of pulsatile ocular blood flow values} between Indians in England and Europeans (study 2)

Table 2 shows the comparison of mean values of age, spherical refraction, average IOP measured using the POBF Tonograph, pulse amplitude, pulse rate, and POBF in Indians and Europeans at Anglia Polytechnic University. The groups were comparable for age (one-way ANOVA, $F=2.3 ; P>0.05$ ), mean spherical refraction (one-way ANOVA, $F=0.20 ; P>0.05$ ), and IOP measured by the POBF Tonograph (one-way ANOVA, $F=0.32 ; P>0.05)$.

The difference in POBF between the two groups did not reach statistical significance (one-way ANOVA, $F=0.17 ; P>0.05)$. Females had a higher mean POBF than males in both Indians in England and European groups (one-way ANOVA, $F=13.29 ; P<0.001$ ) but there was no interaction due to ethnic differences (two-way ANOVA, $F=1.09 ; P>0.05)$.

\section{Comparison of POBF of Indians with Europeans and Indians in England}

In comparing the mean values of POBF in the two geographical regions, we found that there was no significant difference in mean spherical refraction (one-way ANOVA, $F=0.08 ; P>0.05$ ) and average IOP measured by POBF Tonograph (one-way ANOVA,

Table 1 Pulsatile ocular blood flow reference values for Indians

\begin{tabular}{lccc}
\hline Mean & Males & Females & Mean of males and females $(S D$, range) \\
\hline Age (years) & 34.00 & 31.99 & $32.96(14.4,13-73)$ \\
Refraction (D) & -0.80 & -0.42 & $-0.60(2.1,-12.75-+4.50)$ \\
Goldmann IOP (mmHg) & 14.5 & 14.9 & $14.7(2.42,9-21)$ \\
POBFT IOP (mmHg) & 14.66 & 14.49 & $14.57(3.59,14.13-15.02)$ \\
Pulse rate/min ${ }^{\mathrm{a}}$ & 73.5 & 77.3 & $75.5(11.20,50-115)$ \\
Pulse amplitude (mmHg) & 2.93 & 3.40 & $3.17(1.16,1-11)$ \\
Pulsatile ocular blood flow $(\mu \mathrm{l} / \mathrm{min})$ & 1052 & 1293 & $1176(402.8,320-2588)$ \\
\hline
\end{tabular}

$\mathrm{POBFT}=$ pulsatile ocular blood flow tonograph; $\mathrm{IOP}=$ intraocular pressure; $\mathrm{SD}$ is standard deviation .

${ }^{a}$ Measured by the pulsatile ocular blood flow tonograph.

Table 2 Comparison of pulsatile ocular blood flow values between Indians in England and Europeans

\begin{tabular}{lcc}
\hline & \multicolumn{2}{c}{ Mean (SD, range) } \\
\cline { 2 - 3 } & Indian in England & European \\
\hline Age (years) & $23.3(4.6,18-38)$ & $25.0(5.3,18-37)$ \\
Refraction $(\mathrm{D})$ & $-0.74(1.1,-4.00-0.75)$ & $-0.63(1.2,-4.00-0.00)$ \\
POBFT IOP $(\mathrm{mmHg})$ & $15.65(3.6,7.5-22.3)$ & $15.14(4.0,10.1-22.7)$ \\
Pulse rate/min $\mathrm{m}_{\text {a }}$ & $75.5(13.04,56-113)$ & $73.8(14.19,45-115)$ \\
Pulse amplitude $(\mathrm{mmHg})$ & $2.9(0.88,1.4-5.1)$ & $3.1(0.97,1.6-6.5)$ \\
Pulsatile ocular blood flow $(\mu \mathrm{l} / \mathrm{min})$ & $1061(323.4,523-1761)$ & $1033(289.6,465-1580)$ \\
\hline
\end{tabular}

POBFT $=$ pulsatile ocular blood flow tonograph; IOP = intraocular pressure.

${ }^{\mathrm{a}}$ Measured by the pulsatile ocular blood flow tonograph. 
$F=1.70 ; P>0.05)$ of the different groups; however, there was a significant difference in mean age of the groups (one-way ANOVA, $F=14.70 ; P<0.0001$ ) with the mean age of the Indian group being greater than the European and Indians in England groups. Because there was a significant difference in mean age within groups, a one-way ANCOVA was used to investigate the difference in POBF with age as the covariate. Accordingly, the analyses revealed that age differences were not the cause of the significantly higher mean POBF for the Indian group relative to the European sample (one-way ANCOVA, $F=4.70 ; P<0.05)$ and Indians in England group (one-way ANCOVA, $F=3.44 ; P<0.05$ ).

\section{Discussion}

The main result of this study is that, while Europeans and people of Indian ethnic origin living in England have similar POBF values, Indians living in India have slightly higher POBF values. However, the POBF values of all groups measured in the present study are substantially higher than in previous reports. . $^{8,10,11,20-22}$

Table 3 shows the published values for mean POBF compared with the results of the present study. All studies in Table 3 involve normal subjects, with POBF measured, while subjects were in a seated position with the POBF Tonograph mounted on a slitlamp. Our finding that females had higher POBF values than males is consistent with previous reports ${ }^{10,21,22}$ and is likely to be due to the hormonal and ocular volume differences between males and females. ${ }^{10,21,22}$ Also females tend to have higher pulse rate. ${ }^{10,21,23}$ The finding of a difference in pulse rate between males and females in the current study, as measured by the POBF Tonograph, is similar to the findings of previous reports and may be a partial explanation of the difference in POBF values between males and females. ${ }^{10,21,23}$ In the present study, age had no effect on POBF values although a negative relationship with age has previously been reported. ${ }^{21,22}$

Refractive error influences POBF values, with more myopic refractive errors producing lower POBF values, confirming the reports of previous investigations. ${ }^{8,22,24}$ It has been proposed that the influence of refractive error on POBF relates to axial length ${ }^{20,23,24}$ as mypoes tend to have greater ocular volume, choroidal thinning, and greater scleral elasticity. The median spherical equivalent refractive error in the male and female subjects reported here was $0.00 \mathrm{D}$ (range -12.75 to $+4.50 \mathrm{D}$ ). Axial length was not measured in the present study, but the axial length is expected to be longer in myopes and shorter in hypermetropes. Since the median spherical error in the three ethnogeographical groups in the present study was emmetropia, axial length, and refractive error were considered unlikely causes of the much higher mean POBF values found in the present study compared with those in previous studies. . $^{80,11,20-22}$

Because POBF is indirectly measured using IOP pulse amplitude, heart rate differences, and blood pressure could influence it. Also blood pressure lowering medications could lower POBF and substances like nicotine and caffeine could increase blood pressure and thus increase POBF. Blood pressure was not measured in the present study; however, the subjects involved in the present studies did not have a known history of any systemic disease or a regular intake of any medications. Therefore, we feel that cardiovascular disease is unlikely to be an explanation for the difference in results.

Lam $e t a l^{8}$ found relatively low POBF values in Hong Kong Chinese. This was attributed to refractive error, there being a high prevalence of myopia within the Hong Kong Chinese, although other racial and genetic influences could not be excluded.

The ethnicity of subjects in the studies of Morgan and Hosking, ${ }^{9}$ Yang et $a l,{ }_{10}^{10}$ Fontana et $a l,{ }_{11}^{11}$ and Gekkieva $e t ~ a l^{21}$ are not known, but the subjects are presumed to be mainly European, since the studies were all conducted in Europe.

The POBF Tonograph used in the present study was calibrated according to the manufacturer's instructions, and gave IOP values comparable with those obtained by Goldmann applanation tonometry. We find it difficult to attribute the much higher POBF values found in the present study to actual differences in POBF between our

Table 3 Comparison of the mean pulsatile ocular blood flow reported in different studies

\begin{tabular}{|c|c|c|c|c|}
\hline Study & Total subjects & $\operatorname{POBF}(\mu \mathrm{l} / \mathrm{min})$ & Instrument & Male:female \\
\hline Massey et $a l^{22}$ & 664 & 808 (Mean POBF) & OBF system (Software version unknown) & Not known \\
\hline Yang et $a l^{10}$ & 167 & 756 (Mean POBF) & OBF system (Software version unknown) & $73: 94$ \\
\hline Fontana et $a l^{11}$ & 777 (1554 eyes) & 824 (Median POBF) & OBF system (Software version 3.01 ) & $347: 430$ \\
\hline Gekkieva et $a l^{21}$ & 155 & 685 (Mean POBF) & Langham Blood Flow system & $86: 69$ \\
\hline Mori et al ${ }^{20}$ & 80 & 593.3 (Median POBF) & Langham Blood Flow system & $27: 53$ \\
\hline Lam $e t a l^{8}$ & 74 & 661.2 (Mean POBF) & OBF system (Software version unknown) & $33: 41$ \\
\hline Morgan et $a l^{9}$ & 16 & 1198 (Mean POBF) & OBF system (Software version unknown) & Not known \\
\hline $\begin{array}{l}\text { Indians } \\
\text { (Present study in India) }\end{array}$ & 252 & 1176.3 (Mean POBF) & OBF system (Software version 16.2) & $122: 130$ \\
\hline
\end{tabular}


European subjects and those who took part in previous studies. Morgan and Hosking ${ }^{9}$ found similar POBF values to those reported here and pointed out that there is no 'gold standard' for POBF measurement.

We believe that the large differences between studies (Table 3 except Morgan et al), are more likely due either to a difference in the sensor construction which affects the pulse measurement more than the IOP measurement, or to a difference in the software used to calculate POBF in different versions. We cannot rule out, from our results, that small differences in operator procedures may be the cause of our higher values, however a previous study reported good interobserver reliability ${ }^{8}$ for POBF measurement.

There was no significant difference between mean POBF of the Indians in England when compared to the European group. This suggests that when the methodology and instrumentation are standardized and other variables such as age, mean spherical refraction, and IOP are accounted for, there was no difference in POBF between Europeans and Indians in England. To detect a clinically significant difference of $250 \mu \mathrm{l} / \mathrm{min}$ (as reported by other study ${ }^{10}$ ) the present study had a power of 0.95 (one-tailed $t$-test, effect size $=0.74$ ).

Comparing the three groups, we found that the POBF in the study in India was slightly higher than for the Europeans and the Indians in England. Additionally, all groups were comparable for IOP and refraction. The difference in POBF persisted despite accounting for age which was found to be significantly different between groups. One can hypothesize that such small differences in mean POBF between the residents of India and England could arise due to environmental factors such as ambient temperature and differences in atmospheric pressure (although POBF machine used in the present study has a pressure calibration device). Also the differences in body mass index and height could influence POBF as they are independently correlated with ocular dimensions like axial length. ${ }^{25,26}$

Finally, the results of the present study provide a normative database for future studies in Indian populations if the same version of POBF Tonograph is used, and imply that small differences in the measured POBF of different studies could arise due to environmental factors.

\section{Acknowledgements}

We thank the chairman of Medical Research Foundation Chennai India, Dr SS Badrinath and our beloved late Dr Srinivas, Principal Elite, School of Optometry, Chennai, India for providing the necessary infrastructure for the study.

\section{References}

1 Silver DM, Farrell RA. Validity of pulsatile ocular blood flow measurements. Surv Ophthalmol 1994; 38 (Suppl): S72-S80.

2 Krakau CE. Calculation of the pulsatile ocular blood flow. Invest Ophthalmol Vis Sci 1992; 33: 2754-2756.

3 Krakau CE. A model for pulsatile and steady ocular blood flow. Graefe's Arch. Clin Exp Ophthalmol 1995; 233: 112-118.

4 Moses R, Grodzki W. The pneumotonograph - a laboratory study. Arch Ophthalmol 1979; 97: 547-552.

5 Phillips CI, Tsukahara S, Hosaka O, Adams W. Ocular pulsation correlates with ocular tension: the choroid as piston for an aqueous pump? Ophthalmic Res 1992; 24: 338-343.

6 Quigley HA, Langham ME. Comparative intraocular pressure measurements with the pneumotonograph and goldmann tonometer. Am J Ophthalmol 1975; 80: 266-273.

7 Silver DM, Farrell RA, Langham ME, O'Brien V, Schilder P. Estimation of pulsatile ocular blood flow from intraocular pressure. Acta Ophthalmol Suppl 1989; 191: 25-29.

8 Lam AK, Chan H, Fan W, To CH. A preliminary study on the ocular blood flow (OBF) of Hong Kong Chinese. Ophthalmic Physiol Opt 1999; 19: 512-517.

9 Morgan A, Hosking S. Ocular blood flow tonometer reproducibility: the effect of operator experience and mode of application. Ophthalmic Physiol Opt 2001; 21(5): 401-406.

10 Yang YC, Hulbert MF, Batterbury M, Clearkin LG. Pulsatile ocular blood flow measurements in healthy eyes: reproducibility and reference values. J Glaucoma 1997; 6: 175-179.

11 Fontana L, Poinoosawmy D, Bunce CV, O’Brien C, Hitchings RA. Pulsatile ocular blood flow investigation in assymetric normal tension glaucoma and normal subjects. Br J Ophthalmol 1998; 82: 731-736.

12 James CB, Smith SE. Pulsatile ocular blood flow in patients with low tension glaucoma. Br J Ophthalmol 1991; 75: 466-470.

13 Langham ME. Ocular blood flow and vision in healthy and glaucomatous eyes. Surv Ophthalmol 1994; 38 (Suppl): S161-S168.

14 Quaranta L, Manni G, Donato F, Bucci MG. The effect of increased intraocular pressure on pulsatile ocular blood flow in low tension glaucoma. Surv Ophthalmol 1994; 38 (Suppl): S177-S181; discussion S182.

15 Ravalico G, Pastori G, Toffoli G, Croce M. Visual and blood flow responses in low-tension glaucoma. Surv Ophthalmol 1994; 38 (Suppl): S173-S176.

16 MacKinnon JR, O'Brien C, Swa K, Aspinall P, Butt Z, Cameron D. Pulsatile ocular blood flow in untreated diabetic retinopathy. Acta Ophthalmol Scand 1997; 75: 661-664.

17 Schmidt KG, von Ruckmann A, Kemkes-Matthes B, Hammes HP. Ocular pulse amplitude in diabetes mellitus. Br J Ophthalmol 2000; 84: 1282-1284.

18 Chen SJ, Cheng CY, Lee AF, Lee FL, Chou JC, Hsu WM et al. Pulsatile ocular blood flow in asymmetric exudative age related macular degeneration. Br J Ophthalmol 2001; 85: 1411-1415.

19 Hessemer V, Schmidt KG. Influence of panretinal photocoagulation on the ocular pulse curve. Am J Ophthalmol 1997; 123: 748-752.

20 Mori F, Konno S, Hikichi T, Yamaguchi Y, Ishiko S, Yoshida A. Pulsatile ocular blood flow study: decreases in 
exudative age related macular degeneration. $\mathrm{Br} J$ Ophthalmol 2001; 85: 531-533.

21 Gekkieva M, Orgul S, Gherghel D, Gugleta K, Prunte C, Flammer J. The influence of sex difference in measurements with the Langham Ocular Blood Flow System. Jpn J Ophthalmol 2001; 45(5): 528-532.

22 Massey AD, O'Brien C. Pulsatile ocular blood flow: population study of normals. Invest Ophthalmol Vis Sci 1996; 36: 136.

23 James CB, Trew DR, Clark K, Smith SE. Factors influencing the ocular pulse - axial length. Graefes Arch Clin Exp Ophthalmol 1991; 229: 341-344.
24 Ravalico G, Pastori G, Croce M, Toffoli G. Pulsatile ocular blood flow variations with axial length and refractive error. Ophthalmologica 1997; 211: 271-273.

25 Saw SM, Chua WH, Hong CY, Wu HM, Chia KS, Stone RA et al. Height and its relationship to refraction and biometry parameters in Singapore Chinese children. Invest Ophthalmol Vis Sci 2002; 43(5): 1408-1413.

26 Wong TY, Foster PJ, Johnson GJ, Klein BE, Seah SK. The relationship between ocular dimensions and refraction with adult stature: the Tanjong Pagar Survey. Invest Ophthalmol Vis Sci 2001; 42(6): 1237-1242. 